\title{
Deux voix, deux pensées puissantes
}

Two Voices, Two Powerful Minds

Dos voces, dos pensamientos fuertes

Rose-Marie Lagrave

\section{(2) OpenEdition}

Journals

Édition électronique

URL : http://journals.openedition.org/bssg/139

DOI : 10.4000/bssg.139

ISSN : 2490-9424

Éditeur

Presses universitaires de Vincennes

Référence électronique

Rose-Marie Lagrave, "Deux voix, deux pensées puissantes », Biens Symboliques / Symbolic Goods [En ligne], 1 | 2017, mis en ligne le 15 octobre 2017, consulté le 04 mars 2021. URL : http://

journals.openedition.org/bssg/139; DOI : https://doi.org/10.4000/bssg.139 

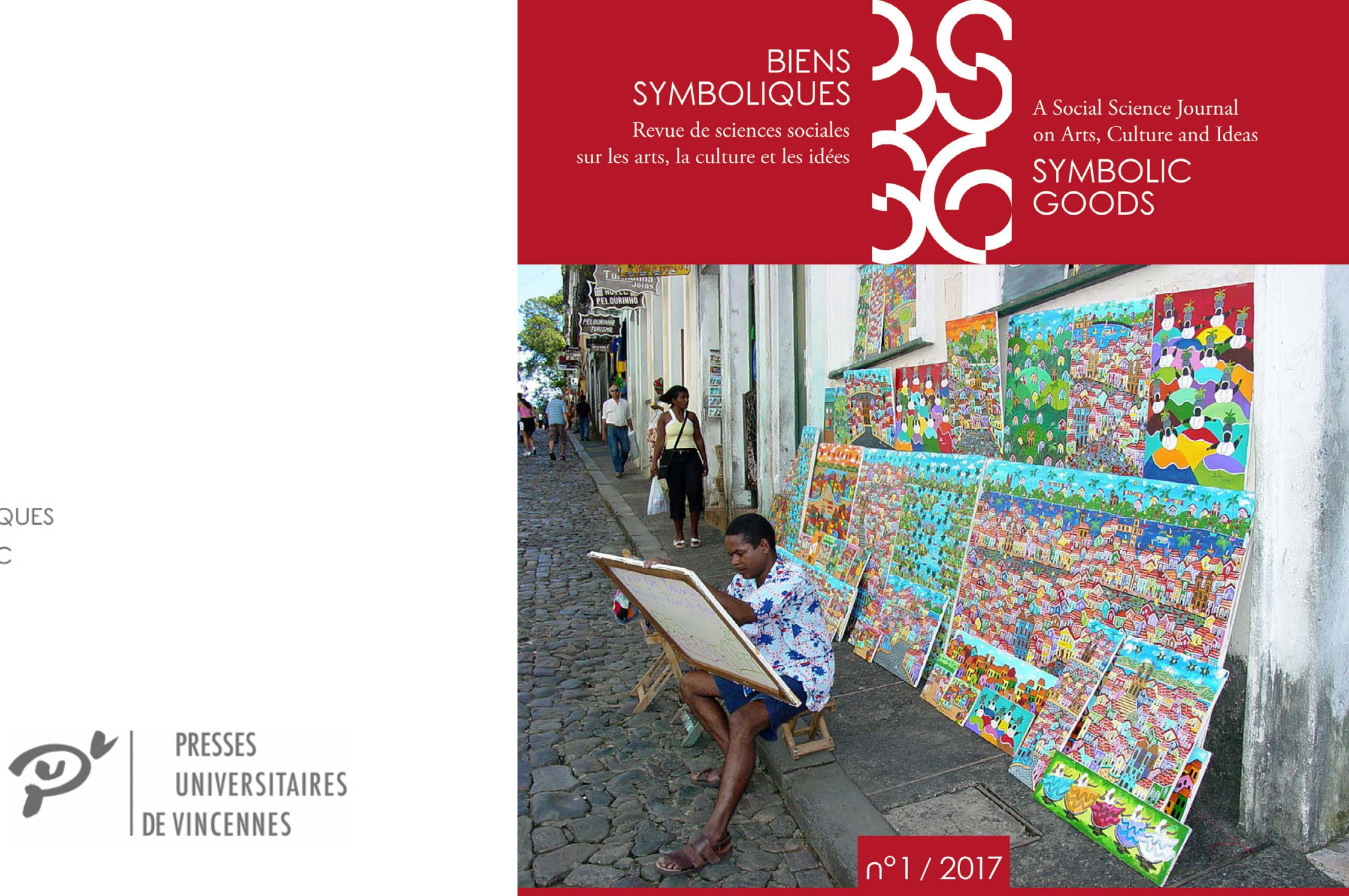

\section{Artistes ordinaires \\ Ordinary Artists}




\title{
Deux voix, deux pensées puissantes
}

\author{
Rose-Marie Lagrave
}

Faire un retour réflexif sur ce moment d'élaboration de l'ouvrage Le Savant et le Populaire que fut le séminaire de l'année 1982 suscite en moi un trouble sentiment à l'égard des souvenirs d'alors, et à l'égard de la position que je suis censée prendre à présent sur le dessein et les effets méthodologiques ultérieurs de ce livre. Car j'étais mal à l'aise dans ce séminaire, malaise au regard des propositions avancées dans cet ouvrage, et plus encore mal à l'aise dès que l'on parle de culture populaire. Et pourtant, il me faut avouer que ce séminaire a déstabilisé mes manières de faire de la sociologie, alors que je n'endosse pas complètement le canevas légitime construit par Grignon et Passeron. Cet état d'esprit paradoxal est sociologiquement explicable dès lors que l'on tente d'objectiver en miroir les raisons d'une aversion pour «le populaire » et les raisons d'une gêne sociale lors des séances de ce séminaire. Cette tension paradoxale a orienté ma lecture de la « littérature paysanne », et constitué le ressort sur lequel j'ai construit mon rapport à l'Association des Écrivains-Paysans (AEP). Si cette expérience à la fois sociale et scientifique n'a jamais été traduite en termes de rapport au «populaire », il convient néanmoins d'interroger cet évitement de façon réflexive pour mettre au jour la part d'inconscient social dû à ma position sociale d'origine, et la part proprement scientifique qui réfute le point de vue légitimiste, dès lors qu'il s'octroie le droit de qualifier de populaire tout ce qui ne ressort pas de son périmètre. Je me souviens d'un séminaire à deux voix qui se cherchaient, s'écartaient, se croisaient, et bataillaient en une joute oratoire qui résonnait tel un match de tennis. Je me souviens de la polyphonie des deux officiants à mesure du souffle de leur pensée, des scansions de leurs arguments arrimés les uns aux autres, et de la rythmique donnée à un travail en train de s'élaborer sous nos yeux. Les séances donnaient matière à une conversation au sens noble du terme entre deux sociologues rompus à travailler ensemble, à partir de schèmes théoriques et méthodologiques partagés, décidés à soumettre à la critique les conclusions de La Distinction (Bourdieu 1979). L'impression me reste qu'ils parlaient pour eux, entre eux, par spectre de Bourdieu interposé, et que nous, jeunes chercheur.e.s dans l'auditoire, servions de miroir réfléchissant, renvoyant ou relançant leur dialogue. Le rythme effréné de leur pensée suscitait le quasi silence du public, comme en atteste dans l'ouvrage la rareté des prises de paroles (au nombre de neuf) lors de ce séminaire. Ces interventions avaient ainsi moins pour but d'insérer les questions du public dans la fabrique du Savantet [du] Populaire, que de témoigner qu'il s'agissait bien d'un séminaire. Elles font figure de faire-valoir ou de pause pour relancer une idée. Ce séminaire était traversé par une hiérarchie de légitimités, au 
sein de laquelle Grignon et Passeron discutaient en pensant, tandis que le public discutait leur pensée. Les effets de la domination symbolique étaient à l'œuvre dans l'espace du séminaire, mais nul n'y prêtait attention puisqu'en principe, elle ne concernait que l'espace de la littérature. Pourtant, la domination symbolique jouait entre les deux professeurs et les auditeurs, et entre les auditeurs pareillement. Dans mon souvenir, corrigé par les noms mentionnés dans le livre, les anciens étudiants ou collaborateurs de JeanClaude Passeron composaient le gros des troupes ${ }^{1}$. Tel n'était pas mon cas, même si j'avais été son étudiante en licence de sociologie, lorsque Passeron était assistant de Raymond Aron à la Sorbonne. Sa rigueur méthodologique, son raisonnement en "scolies », et jusqu'à sa redingote noire, très Troisième République, m'impressionnaient déjà. La familiarité des jeunes chercheur.e.s avec le travail de Passeron m'a tout de suite donné le sentiment d'une mise à l'écart. Je me sentais comme une sorte de «petit chose » au féminin (Daudet 1868) fourvoyé dans la cour des grands - cour masculine de surcroît. À la faveur des enseignements de Pierre Bourdieu, j'avais déjà pris conscience que mon doctorat, dirigé par Placide Rambaud - à la trajectoire peu légitime selon la définition universitaire (Lagrave 2009) constituait certes un moment décisif d'une ascension sociale inespérée, mais sans pour autant me donner les clefs des codes académiques. La publication de l'ouvrage issu de ma

1 F. de Singly a soutenu sa thèse en 1973 sous la direction de J.-C. Passeron, et M. Grumbach cosignera avec J.-C. Passeron L'CEil à la page : enquête sur les images et les bibliothèques, au Centre Pompidou (1985). En revanche, je n'ai retrouvé aucune trace de P. Parmentier ni de P. Cohen-Scali. thèse, Le Village romanesque (Lagrave 1980), me donna juste ce qu'il faut d'assurance pour participer à ce séminaireassurance bien vite défaite puisque je ne m'étais pas posé les « bonnes » questions. La « littérature paysanne » me semblait emblématique des cas susceptibles d'explorer la tension entre savant et populaire, de sorte que, confiante, curieuse et désireuse de progresser, j'investissais ce séminaire comme une sorte de planche de salut. II le fut à plus d'un titre, tout en se transformant en examen de passage, concernant tant mes connaissances que ma trajectoire sociale.

Dans mon parcours professionnel, le séminaire de 1982 fut l'un de ces moments de remise à ma place, où l'ascension sociale revient en boomerang pour signifier que vous êtes déplacée, à une place qui ne compte pas, à une place que je n'aurais jamais dû occuper. Les deux enseignants, héritiers de Bourdieu quoique volontiers hérétiques, et leur public d'affiliés composaient un cercle enchanté dans lequel j'avais l'impression d'incarner la figure du « populaire » en transition sociale ascendante (Pasquali 2014), éternelle boursière méritante (Lagrave 2010). Médusée et larguée je fus, tant la distance avec mon propre travail se creusait à mesure des séances, tant l'enjeu théorique et méthodologique m'apparaissait encore obscur. Et cette décentration, inséparablement sociale et scientifique, s'est traduite par la hardiesse de poser trois questions qui tombent à plat, incompréhensibles d'ailleurs à la relecture, mais signe toutefois que j'avais osé les poser (Grignon \& Passeron $1989: 97,107,138$ ).

Ces questions retraduites à l'écrit attestent une forme de protestation concernantl'analyse de la place de Georges Navel 
(Navel 1945) dans le contexte littéraire de son temps, et d'un appel à prendre au sérieux, par exemple, les correspondances d'ouvriers (Rancière 1981). Où classer Georges Navel, publié chez Gallimard, préfacé par Giono, et ami de Bernard Groethuyssen ? Dans la littérature « populaire » - parce qu'autodidacte, bien qu'ayant suivi les cours de l'Université syndicale - ou parmi les écrivains légitimes ? Ma volonté, je m'en souviens, était d'obliger Grignon et Passeron à venir sur le terrain d'exemples empiriques, et de montrer que l'ambivalence de la position de Georges Navel participant des deux mondes, était un cas emblématique qui déjouait les classifications et les classements opérés par les deux démarches opposées.

Ces questions posées et celles pendantes témoignent d'un essai infructueux pour revenir à des exemples empiriques mettant à l'épreuve les approches proposées. À chaque question, je fus renvoyée dans les cordes, dans une position d'avocate de la « cause du peuple », par des réponses qui détournent ou contournent la question pour poursuivre, sans attendre, la logique du séminaire. Cette condescendance bienveillante à laquelle le milieu académique m'avait habituée fut une opportune provocation pour clarifier dans quel camp méthodologique je me situais, entre populisme et misérabilisme - travail d'élucidation au demeurant impossible à faire sur le champ, mais qui ne laissait pas de me tarauder. La panique mentale éprouvée lors de ce séminaire fit promptement place à une remise sur le métier du Village romanesque pour tenter, en bonne élève, d'appliquer les enseignements du séminaire et de préciser les raisons d'un passage à l'écriture d'autodidactes paysans peu attendus sur la scène littéraire. En passant aux travaux pratiques, avec en tête l'injonction de ne dériver ni vers le populisme, ni vers le misérabilisme, tout en rendant agissante la matrice de la domination sociale et symbolique, j'ai alors procédé à l'examen critique de cet ouvrage. Toutefois, à la relecture de l'article qui s'en est suivi (Lagrave 1988), force est de constater que je ne m'insère nullement dans la tension entre populisme et misérabilisme, tout en utilisant certains enseignements de ce séminaire. On remarque, en effet, un évitement de cette tension méthodologique qui se traduit par une sorte de louvoiement et de ruse pour à la fois saisir le rapport social - l'humiliation culturelle - à partir duquel se fonde la littérature paysanne, pour déconstruire la revendication de ces écrivains à l'authenticité, et pour mettre au jour les rapports de force et les distinctions, à l'intérieur d'un espace littéraire dominé. Ce séminaire de 1982 fut ainsi tout à la fois un moment de bifurcation sociale d'une transition de classe inachevée, et de réévaluation de mon travail sur la littérature paysanne. En ce sens, il a constitué l'une des épreuves pour construire, de façon fragile et incertaine, un itinéraire social et professionnel plus légitime (Lagrave 2010). Mais simultanément, ces séances ont été un rappel à l'ordre légitime, censé se traduire par le recours à une approche sociologique croisant alternance et ambivalence (Grignon \& Passeron 1989, chapitre 2). Ce séminaire fut dès lors une école de modestie et un défi pour retravailler une recherche antérieure.

En reprenant Le Savant et le Populaire pour relire les articles concernant la littérature paysanne que j'ai publiés ultérieurement, je n'ai pas tranché entre la dérive misérabiliste et le penchant populiste, pour mettre au jour les paradoxes 
dans lesquels les chercheur.exs sont prisxes dès qu'ils et elles confrontent ces approches à des cas empiriques. En procédant par touches successives de déconstruction/ reconstruction, on ne parvient toutefois pas à proposer une alternative claire et robuste. Cette contribution témoigne donc de l'état des réflexions suscitées par cette relecture, sans toutefois atteindre l'ambition souhaitée. Le Savant et le Populaire reste, dès lors, un défi pour une recherche plus armée et plus affinée.

\section{Références bibliographiques}

ARON Raymond (1983). Mémoires. Paris, Julliard.

BAKHTINE Mikhaïl (1970). L'CEuvre de François Rabelais et la culture populaire au Moyen Âge et sous la Renaissance. Paris, Gallimard.

BECKER Howard (1988). Les Mondes de l'art. Traduit de l'anglais par Jeanne Bouniort. Paris, Flammarion.

Bellavance Guy, Boivin Micheline, SanterRe Lise (dir.) (2000). Démocratisation de la culture ou démocratie culturelle ? Deux logiques d'action publique. Québec, Éditions de l'IQRC.

Bols Géraldine (2008). "Le consentement à la domination littéraire. Degrés et diversité de ses formes chez les écrivains "les moins reconnus" de l'univers littéraire ». Tracés, $14:$ 55-76.

Bourdieu Pierre (1979). La Distinction. Critique sociale du jugement. Paris, Minuit.

BoURDIEU Pierre (1984). Questions de sociologie. Paris, Minuit.

BouRdieu Pierre (1988). «Entretien recueilli par Beate Krais ». In BouRdieU Pierre, Chamboredon Jean-Claude, Passeron Jean-Claude, Le Métier de sociologue. Préalables épistémologiques. Paris, Éditions de l'EHESS (cinquième édition)

BouRdieu Pierre (1989). La Noblesse d'État. Paris, Minuit.

BouRdieu Pierre (1993). « Les contradictions de l'héritage ». In Bourdieu Pierre (dir.), La Misère du monde. Paris, Seuil : 711-718.
BOURDIEU Pierre (1998) [1992]. Les Règles de l'art. Genèse et structure du champ littéraire. Paris, Seuil.

BouRdieu Pierre (1998). La Domination masculine. Paris, Seuil.

Bourdieu Pierre (2003) [1997]. Méditations pascaliennes. Paris, Seuil.

Bourdieu Pierre, Chamboredon Jean-Claude, Passeron Jean-Claude (1968). Le Métier de sociologue. Paris, Mouton.

Bourdieu Pierre \& Passeron Jean-Claude (1964). Les Héritiers. Les étudiants et la culture. Paris, Minuit.

BOURDIEU Pierre \& PASSERON Jean-Claude (1970). La Reproduction. Éléments pour une théorie du système d'enseignement. Paris, Minuit.

BOURDIEU Pierre \& WACQUANT Loïc (2014). Invitation à la sociologie réflexive. Paris, Seuil.

BOUTIER Jean (2008). "Quelques réflexions rétrospectives sur les histoires de la "culture populaire" ». Journée d'étude "Le Savant et le Populaire, 1989-2008. Retour sur un débat en suspens ». SHADYC, EHESS Marseille (non publié).

BouvERESSE Jacques (2008). " Le besoin de croyance et le besoin de vérité ». Agone, 38-39: 281-306.

BURKE Peter (1978). Popular Culture in Early Modern Europe. Londres, Temple Smith.

Buscatto Marie (2014). Sociologies du genre. Paris, Armand Colin.

Busino Giovanni (2006). "Entretien avec Dominique Schnapper ». Revue européenne des sciences sociales, XLIV(135). [En ligne] http://ress. revues.org/271 [consulté le 12 octobre 2016].

Casanova Pascale (2008) [1999]. La République mondiale des lettres. Paris, Seuil.

ChAPOULIE Jean-Michel (1991). " La seconde fondation de la sociologie française, les États-Unis et la classe ouvrière ». Revue française de sociologie 32(3) : 321-364.

CHARTIER Roger (2001). "Culture écrite et littérature à l'âge moderne ". Annales. Histoire, Sciences sociales, 56(4) : 783-802.

COHEN Déborah (2010). La Nature du peuple. Les formes de l'imaginaire social (XVIII/XXP siècles). Seyssel, Champ Vallon.

COLLECTIF (1988). « Histoire et sciences sociales. Un tournant critique ? ». Annales ESC, 2 : 291-293. 
Collectif Révoltes logiques (1984). L'Empire du sociologue. Paris, La Découverte.

Collovald Annie (1988). «Identité(s) stratégiques(s) », Actes de la recherche en sciences sociales, $73: 29-40$.

Connell Raewyn (1995). Masculinities. Cambridge, Polity Press.

CoRnu Tanguy (2008). « L'ambiguïté du concept de domination symbolique

dans Le Savant et le Populaire ". Journée d'étude "Le Savant et le

Populaire, 1989-2008. Retour sur un débat en suspens ". SHADYC, EHESS Marseille (non publié).

CoulangeOn Philippe \& Duval Julien (2013). Trente ans après La Distinction de Pierre Bourdieu. Paris, La Découverte.

COULANGEON, Philippe \& DUVAL Julien (2013). «Introduction ». In EID. (dir.) Trente ans après La Distinction de Pierre Bourdieu. Paris, La Découverte. DAUDET Alphonse (1868). Le Petit Chose. Paris, Pierre-Jules Hetzel.

DebeauvaIS Michel (1976). L'Université ouverte : les dossiers de Vincennes. Grenoble, Presses Universitaires de Grenoble.

Dragomir Lucia (2011). " L'Union des écrivains. Un modèle institutionne et ses limites ». Vingtième siècle, $109: 59-70$.

Ducournau Claire (2017). "Les deux (ou trois) carrières de Richard Hoggart. De la fondation des cultural studies aux appropriations de la sociologie française ». Revue d'anthropologie des connaissances, 11(3) 263-282.

DUMONT Fabienne \& SoFIO Séverine (2007). "Esquisse d'une épistémologie de la théorisation féministe en art ». Cahiers du genre, 43 : 17-43.

DUVAL Julien (2010). "Distinction studies ». Actes de la recherche en sciences sociales, 181-182: 146-156.

ELIAS Norbert (1991). La Société des individus. Traduit de l'allemand par Jeanna Etoré. Paris, Fayard.

FABIANI Jean-Louis (dir.) (2001). Le Goût de l'enquête. Pour Jean-Claude Passeron. Paris, L'Harmattan.

FossÉ-PolıAK Claude (2006). Aux frontières du champ littéraire. Sociologie des écrivains amateurs. Paris, Économica.

Foucault Michel (1973). Moi, Pierre Rivière ayant égorgé ma mère, ma sœur et mon frère... Paris, Gallimard/Julliard.

FoucAult Michel (1994). Dits et écrits, t. 1 et 2. Paris, Gallimard.
GabORIAU Philippe (1995). Le Tour de France et le vélo. Histoire sociale d'une épopée contemporaine. Paris, L'Harmattan.

GabORIAU Philippe (2003). Les Spectacles sportifs. Grandeurs et décadences. Paris, L'Harmattan.

GinzBURG Carlo (1980) [1976]. Le Fromage et les vers. L'univers d'un meunier $d u X V^{e}$ siècle [Il formaggio e $i$ vermi. II cosmo di un mugnaio del Cinquecento, Turin, Einaudi]. Traduit de l'italien par Monique Aymard. Paris, Flammarion.

Grenier Jean-Yves, Grignon Claude, Menger Pierre-Michel (dir.) (2001). Le Modèle et le récit. Paris, Maison des Sciences de l'Homme.

Grignon Claude (1996). "Le savant et le lettré, ou l'examen d'une désillusion ", Revue européenne des sciences sociales, 103 : 81-98.

GRIGNON Claude (2001). " La formalisation et les sciences du récit, le cas de la sociologie ". In GRENIER Jean-Yves, GRIGNON Claude, MeNGer Pierre-Michel (dir.), Le Modèle et le récit. Paris, Maison des Sciences de l'Homme : 7-43.

Grignon Claude \& Kordon Claude (dir.) (2009). Sciences de l'homme et sciences de la nature, essais d'épistémologie comparée. Paris, Maison des Sciences de l'Homme.

GRIGNON Claude \& PASSERON Jean-Claude (1982). Sociologie de la culture et sociologie des cultures populaires. Paris, Documents du GIDES, 4.

Grignon Claude \& PASSERON Jean-Claude (1985). Enquête. Cahiers du CERCOM, 1 (« À propos des cultures populaires »). [En ligne] https:// enquete.reveues.org/2 [consulté le 26 juin 2017].

GriGnon Claude \& PASSERon Jean-Claude (2015) [1989]. Le Savant et le Populaire. Misérabilisme et populisme en sociologie et en littérature.

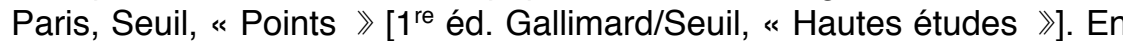
espagnol : Grignon Claude \& PASSERON Jean-Claude (1991). Lo culto y lo popular : miserabilismo y populismo en sociologia y en literatura. Traduction de María Sonderéguer. Buenos Aires, Ediciones Nueva Vision Grignon Claude \& PASSERON Jean-Claude (1992). Lo culto y lo popular miserabilismo y populismo en sociologia y en literatura. Traduction de Fernando Alvarez-Uria et Julia Varela. Madrid, Las Ediciones de La Piqueta. 
Grignon Claude, Collovald Annie, Pudal Bernard, Sawicki Frédéric (1991). « Un savant et le populaire. Entretien avec Claude Grignon ». Politix, $13:$ :35-42.

GRUEL Louis (2004). La Rébellion de 68. Une relecture sociologique. Rennes, Presses Universitaires de Rennes.

GUILLAUMIN Colette (2002) [1972]. L'ldéologie raciste. Paris, Gallimard

HALL Stuart \& JefFERSON Tony (dir.) (1976). Resistance through Rituals : Youth Subcultures in Post-War Britain. Londres, Hutchinson.

Hammou Karim (2008). «Penser le pouvoir avec Le Savant et le Populaire ». Journée d'étude «Le Savant et le Populaire, 1989-2008. Retour sur un débat en suspens ». SHADYC, EHESS Marseille (non publié).

HEINICH Nathalie (2000) [1991]. Être écrivain. Paris, La Découverte.

HoggaRT Richard (1970a). La Culture du pauvre. Étude sur le style de vie des classes populaires en Angleterre. Traduit de l'anglais par Françoise Garcia, Jean-Claude Garcia et Jean-Claude Passeron, présentation et index de Jean-Claude Passeron (titre original The Uses of Literacy Aspects of Working Class Life). Paris, Minuit.

HogGaRT Richard (1970b). Speaking to Each Other, vol. 1. Londres, Chatto et Windus.

HoggaRT Richard (2013) [1991]. 33 Newport Street. Autobiographie d'un intellectuel issu des classes populaires anglaises. Traduit de l'anglais par Christiane Grignon \& Claude Grignon avec la collaboration de Christopher Todd, présentation de Claude Grignon. Paris, Seuil, « Points »

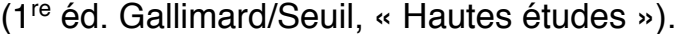

KalIFA Dominique (2005). «Les historiens français et le "populaire" ». Hermès, $42: 54-59$.

KRÉFA Abir (2013). "La quête de l'autonomie littéraire en contexte autoritaire : le cas des écrivains tunisiens ». Sociologie, $4: 395-411$.

KRÉFA Abir (2013). Activités littéraires et rapports sociaux de sexe : le cas des écrivains tunisiens (thèse de doctorat en sociologie). Lyon, Université Lyon 2.

KRÉFA Abir (2014). «Entre injonctions à dire et à taire le corps : les voies étroites de la reconnaissance littéraire pour les écrivaines tunisiennes ». Ethnologie francaise, 44(4) : 631-642.

LAgraVE Rose-Marie (1980). Le Village romanesque. Le Paradou, Actes Sud, « Espace-temps ».
LAGRAVE Rose-Marie (1988). « Mensonge romanesque et vérité des romanciers. Une relecture du Village Romanesque ». Études Rurales $109: 55-73$.

LAGRAVE Rose-Marie (2009). «Filiations intellectuelles et espérance sociale. Figure et œuvre de Placide Rambaud ». Études rurales, 183 : 51-66.

Lagrave Rose-Marie (2010). «Se ressaisir ». Genre, Sexualité \& Société, 4 : 1-17. [En ligne] http://gss.revues.org

LAHIRE Bernard (2004). La Culture des individus : dissonances culturelles et distinction de soi. Paris, La Découverte.

LAHIRE Bernard (2008). La Raison scolaire. École et pratiques d'écriture entre savoir et pouvoir. Rennes, Presses Universitaires de Rennes.

LAHIRE Bernard (2012). Monde pluriel. Penser l'unité des sciences sociales. Paris, La Découverte.

LAMONT Michèle (1995). La Morale et l'argent : les valeurs des cadres en France et aux États-Unis. Paris, Métailié.

LAMONT Michèle (2013). "En quoi Bourdieu a-t-il été utile à notre réflexion ? Le cas des États-Unis ». In CoULANGEON Philippe \& DuvaL Julien (dir.), Trente ans après La Distinction de Pierre Bourdieu. Paris, La Découverte : 59-68.

Levi Giovanni (1989). Le Pouvoir au village. Histoire d'un exorciste dans le Piémont du XvII siècle. Paris, Gallimard.

LINHART Robert (1981). L'Établi. Paris, Minuit.

MACKENZIE Caroline (2012). «Agency, un mot, un engagement ». Rives méditerranéennes, 41(1). [En ligne] http://rives.revues.org/4139 [consulté le 11 mars 2017].

Mathieu Nicole-Claude (1991). L'Anatomie politique. Paris, Côté-Femmes MAUGER Gérard (1991). «Enquêter en milieu populaire ». Genèses, 6 125-143.

MAUGER Gérard (2005). « Un apprentissage tardif du métier de sociologue ». In MAUGER Gérard (dir.), Rencontres avec Pierre Bourdieu. Bellecombe-en-Bauges, Le Croquant : 239-257.

MAUGER Gérard (2006a). "Sur la violence symbolique ». In MüLLER HansPeter \& Sintomer Yves (dir.). Pierre Bourdieu, théorie et pratique. Paris, La Découverte : 84-100. 
MAUGER Gérard (2006b). Les Bandes, le milieu et la bohème populaire. Études de sociologie de la déviance des jeunes des classes populaires (1975-2005). Paris, Belin.

MAUGER Gérard (2013). " Bourdieu et les classes populaires. L'ambivalence des cultures dominées ». In Coulangeon Philippe \& DUVAL Julien (dir.), Trente ans après La Distinction de Pierre Bourdieu. Paris, La Découverte : 243-254.

MAUGER Gérard (2014). « Domination ». Le Lexique socius. [En ligne] http:// ressources-socius.info/index.php/lexique/21-lexique/154-domination [consulté le 14 janvier 2017].

MAUGER Gérard \& FossÉ Claude (1977). La Vie buissonnière. Marginalité petite-bourgeoise et marginalité populaire. Paris, Maspero.

MAUGER Gérard \& Fossé-PoliaK Claude (1985). "Choix politiques et choix de recherches. Essai d'auto-socio-analyse (1973-1984) ». Cahiers " Jeunesses et Sociétés », 3-4-5: 27-121.

MengeR Pierre-Michel (2009). Le Travail créateur. S'accomplir dans l'incertain. Paris, Gallimard/Seuil, « Hautes études ».

Morrison Toni (1990). Playing in the Dark: Whiteness and The Literary Imagination. New York, Vintage Books.

MouLIN Raymonde (1971). "La Culture du pauvre. À propos du livre de Richard Hoggart ». Revue française de sociologie, 12(2) : 255-258.

Moulin Raymonde (1992). L'Artiste, l'institution et le marché. Paris, Flammarion.

MouLIN Raymonde (dir.) (1986). Sociologie del'art. Paris, La Documentation française.

Moulin Raymonde, Passeron Jean-Claude, PAsquier Dominique, PortoVASQUEZ Fernando (1985). Les Artistes, essai de morphologie sociale. Paris, La Documentation Française.

NAUDIER Delphine \& Rollet Brigitte (2007). Genre et légitimité culturelle. Quelle reconnaissance pour les femmes ?. Paris, L'Harmattan.

NAVEL Georges (1945). Travaux. Paris, Gallimard.

Neveu Érik (1999). «Pour en finir avec l'enfantisme. Retours sur enquêtes ". Réseaux, 92-93: 175-201.

Neveu Érik (2008). «Les voyages des cultural studies ». L'Homme, 187$188: 315-341$
NoIRIEL Gérard (1988). Le Creuset français. Histoire de l'immigration (XIXe-XXe siècle). Paris, Seuil.

Olivier DE SARDAN Jean-Pierre (2008). La Rigueur du qualitatif. Les contraintes empiriques de l'interprétation socio-anthropologique. Louvain, Bruylant Academia.

PARETo Vilfredo (1917). Traité de sociologie générale. Traduit de l'italien par Pierre Boven. Lausanne, Payot.

PASQUALI Paul \& SchwaRTZ Olivier (2016). "La Culture du pauvre : un classique revisité. Hoggart, Les classes populaires et la mobilité sociale ». Politix, 114 : 21-45.

Pasqual Paul (2014). Passer les frontières sociales. Comment les " filières d'élite » entrouvrent leurs portes. Paris, Fayard.

PASQUIER Dominique (1989). La Culture des sentiments. L'expérience télévisuelle des adolescents. Paris, Editions de la MSH.

PASQUIER Dominique (2005). " La "culture populaire" à l'épreuve des débats sociologiques ». Hermès, $42: 60-69$.

PASSERON Jean-Claude (2006) [1991]. Le Raisonnement sociologique. Un espace non poppérien de l'argumentation. Paris, Albin Michel, nouvelle édition revue et augmentée (première édition Paris, Nathan).

Passeron Jean-Claude \& Pedler Emmanuel (1991). Le Temps donné aux tableaux. Compte rendu d'une enquête au musée Granet. Marseille, Imerec.

PASSERON Jean-Claude \& GRUMBACH Michel (dir.) (1985). L'CEil à la page : enquête sur les images et les bibliothèques. Paris, Bibliothèque publique d'information, Service des études et de la recherche.

PAsseron Jean-Claude (dir.) (1999). Richard Hoggart en France. Paris, Bibliothèque publique d'information.

PedLeR Emmanuel (2010). « Les sociologies de la musique de Max Weber et Georg Simmel. Une théorie relationnelle des pratiques musiciennes ». L'Année sociologique, 60(2) : 305-330.

Pedler Emmanuel (2016). L'Esprit des lieux. Paris, Éditions de l'EHESS Pedler Emmanuel \& Bourbonnaud David (2002a). « L'offre du festival "in" d'Avignon : effet d'imposition ou stimulation culturelle ? ». Actes du colloque d'Amiens.

Pedler Emmanuel \& Bourbonnaud David (2002b). « La programmation du Festival. Un pacte de confiance entre l'organisation festivalière et son 
public ». In ETHIS Emmanuel (dir.), Avignon : le public réinventé. Paris, La Documentation française : 131-159.

Pedler Emmanuel \& ZerbiB Olivier (2001). Les Nouvelles Technologies à l'épreuve des bibliothèques. Paris, Bibliothèque publique d'information. Peterson Richard A. \& Simkus Albert (1992). " How Musical Tastes Mark Occupational Status Groups ». In LAMONT Michèle \& FouRNIER Marcel, Cultivating differences : Symbolic Boundaries and the Making of Inequality. Chicago, The University of Chicago Press : 152-168.

Peterson Richard A. (1992). « Understanding audience segmentation : From elite and mass to omnivore and univore ». Poetics, 21(4) : 243-258. Peterson Richard A. (1997). «The Rise and Fall of Highbrow Snobbery as a Status Marker ». Poetics, 25(2-3) : 75-92.

Peterson Richard A. \& KeRn Roger M. (1996). « Changing Highbrow Taste : from Snob to Omnivore ». American Sociological Review, 61(5) 900-907.

PUDAL Bernard (1991). « Le populaire à l'encan ». Politix, $13: 53-64$.

RANCIÈRE Jacques (1981). La Nuit des prolétaires. Archives du rêve ouvrier. Paris, Fayard.

RANCIĖRE Jacques (2011). La Leçon d'Althusser. Paris, La Fabrique.

REVEL Jacques (dir.) (1996). Jeux d'échelles. La micro-analyse à l'expérience. Paris, Gallimard/Seuil.

RoBInson Eric E. (1968). The New Polytechnics. The People's Universities. Harmondsworth, Penguin

RoEDIGER David (1991). The Wages of Whiteness : Race and the Making of the American Working Class. New York, Verso.

SAPIRO Gisèle (2013a). « La carrière internationale de La Distinction ». In COULANGEON Philippe \& DUvAL Julien (dir.), Trente ans après La Distinction de Pierre Bourdieu. Paris, La Découverte : 45-58

SAPIRO Gisèle (2013b). « Le champ est-il national ? La théorie de la différenciation sociale au prisme de l'histoire globale ». Actes de la recherche en sciences sociales, $200: 70-85$

SCHILLING Heinz (1981). Konfessionskonflikt und Staatsbildung. Eine Fallstudie über das Verhältnis von religiösem und sozialem Wandel in der Früh Neuzeit am Beispiel der Grafschaft Lippe. Gütersloh, Bertelsmann.

ScotT James (2008). La Domination et les arts de la résistance. Fragments du discours subalterne. Traduction française par Olivier Ruchet. Paris, Éditions Amsterdam.
Servien Pius (1935). Principes d'esthétique. Problèmes d'art et langage des sciences. Paris, Boivin.

SINGLY François (DE) (1998). «Bourdieu : nom propre d'une entreprise collective ». Le Magazine littéraire, 369 : 39-44.

SutTon-Smith Brian (1970). «Psychology of Childlore : The Triviality Barrier ». Western Folklore, 29(1): 1-8.

TAINE Hyppolite (1858). Essais de critique et d'histoire. Paris, Hachette. VeYne Paul (1971). Comment on écrit l'histoire. Essai d'épistémologie. Paris, Seuil.

VeYNe Paul (2008). Foucault. Sa pensée, sa personne. Paris, Albin Michel. Weber Max (1998) [1921]. Sociologie de la musique. Les fondements rationnels et sociaux de la musique [Die rationalen und soziologischen Grundlagen der Musik, Tübingen, Drei Masken Verlag]. Traduit de l'allemand par Jean Molino et Emmanuel Pedler. Paris, Métailié.

WIMMER Andreas \& GLICK SCHILLER Nina (2002). « Methodological Nationalism and Beyond : Nation-State Building, Migration and the Social Sciences ». Global Network, 2 : 301-334. 